Ålesund, Norway; ${ }^{6}$ Hosp. Østfold, Moss, Norway; ${ }^{7}$ Univ. Hosp. of N. Norway, Tromsø, Norway; ${ }^{8}$ Revmatismesykehuset, Lillehammer, Norway; ${ }^{9}$ Martina Hansens Hosp., Bærum, Norway; ${ }^{10} \mathrm{Helgelandssykehuset,} \mathrm{Mo} i$ Rana, Norway; ${ }^{11}$ Brigham and Women's Hosp., Boston, United States of America; ${ }^{12}$ Leiden Univ., Leiden, Netherlands

Background: Remission is the preferred treatment target in rheumatoid arthritis (RA), and many patients require biologic DMARDs to reach this state. It is debated whether tapering of tumor necrosis factor inhibitor (TNFi) treatment to discontinuation should be considered in RA patients who sustain remission on treatment (1).

Objectives: The primary study objective was to assess the effect of tapering and withdrawal of TNFi on the risk of flares in RA patients in clinical remission

Methods: In the non-inferiority ARCTIC REWIND trial, RA patients in remission for at least 12 months on stable TNFi therapy were randomly assigned to continued stable TNFi or tapering (half-dose TNFi for 4 months, thereafter withdrawal of TNFi), with visits every four months. csDMARD co-medication was kept stable in both arms. Patients had to be in DAS remission at inclusion with $0 / 44$ swollen joints. The primary endpoint was the proportion of patients with disease flare during the 12-month study period (defined as DAS $>1.6$, change in DAS $>0.6$ and 2 or more swollen joints, or the physician and patient agreed that a clinically significant flare had occurred). Full-dose TNFi was reinstated in case of flares in the tapering arm. The non-inferiority margin was $20 \%$, with a predefined superiority test if non-inferiority was not shown. The inferiority null-hypothesis was tested in the per-protocol population by mixed effect logistic regression. Radiographs were scored by van der Heijde modified Sharp score (0 and 12 months, average of two readers, progression: $\geq 1$ unit change). Clinicaltrials NCT01881308.

Results: We randomised 99 patients, 92 received the allocated treatment strategy, 84 were included in the per-protocol population. Baseline characteristics, clinical and ultrasound disease activity were balanced (Table). csDMARD co-medication was used by $93 \%$ in the stable and $88 \%$ in the tapering arm. In the primary analysis, $5 \%$ of patients in the stable TNFi arm experienced a flare during 12 months, compared to $63 \%$ in the tapering TNFi arm. The risk difference $(95 \% \mathrm{Cl})$ was $58 \%(42 \%$ to $74 \%$, Fig 1$)$, with stable treatment being deemed superior to tapering. $90 \%$ in the stable and $81 \%$ in the tapering arm did not show progression of radiographic joint damage, difference $(95 \% \mathrm{Cl})-9 \%(-24 \%, 6 \%)$. At 12 months, DAS scores, DAS remission and function were similar between groups (Fig 2). The numbers of adverse events (AE)/serious $A E$ in the stable and tapering arm were 57/2 and 50/3, respectively, with 26 and 15 infections.

Conclusion: In a randomised clinical trial assessing patients in prolonged and deep RA remission, we observed a large increase in the flare rate in patients who tapered and discontinued TNFi. Patients responded well to reinstated treatment and remission rates in the two study arms were comparable at 12 months.

References:

[1] Smolen et al. EULAR recommendations for the management of rheumatoid arthritis with synthetic and biological disease-modifying antirheumatic drugs: 2019 update. ARD 2020

Table. Baseline values $-\mathbf{n}(\%)$, mean (SD), or median (IQR)

\begin{tabular}{lcc}
\hline & Stable, $\mathrm{n}=\mathbf{4 5}$ & Tapering, $\mathrm{n}=\mathbf{4 7}$ \\
\hline Age, yrs & $57(11)$ & $58(13)$ \\
Female & $30(67 \%)$ & $25(53 \%)$ \\
ACPA+ & $35(78 \%)$ & $36(77 \%)$ \\
Symptom duration, yrs & $10(7)$ & $12(7)$ \\
DAS & $0.9(0.4)$ & $0.8(0.3)$ \\
CRP mg/L & $1(1-2)$ & $1(1-3)$ \\
No ulttrasound power Doppler signal in any of 32 joints & $42(96 \%)$ & $44(94 \%)$ \\
\hline
\end{tabular}

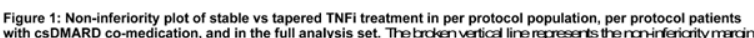

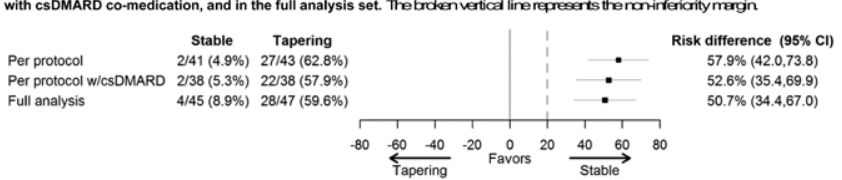

Figure 2: Secondary endpoints
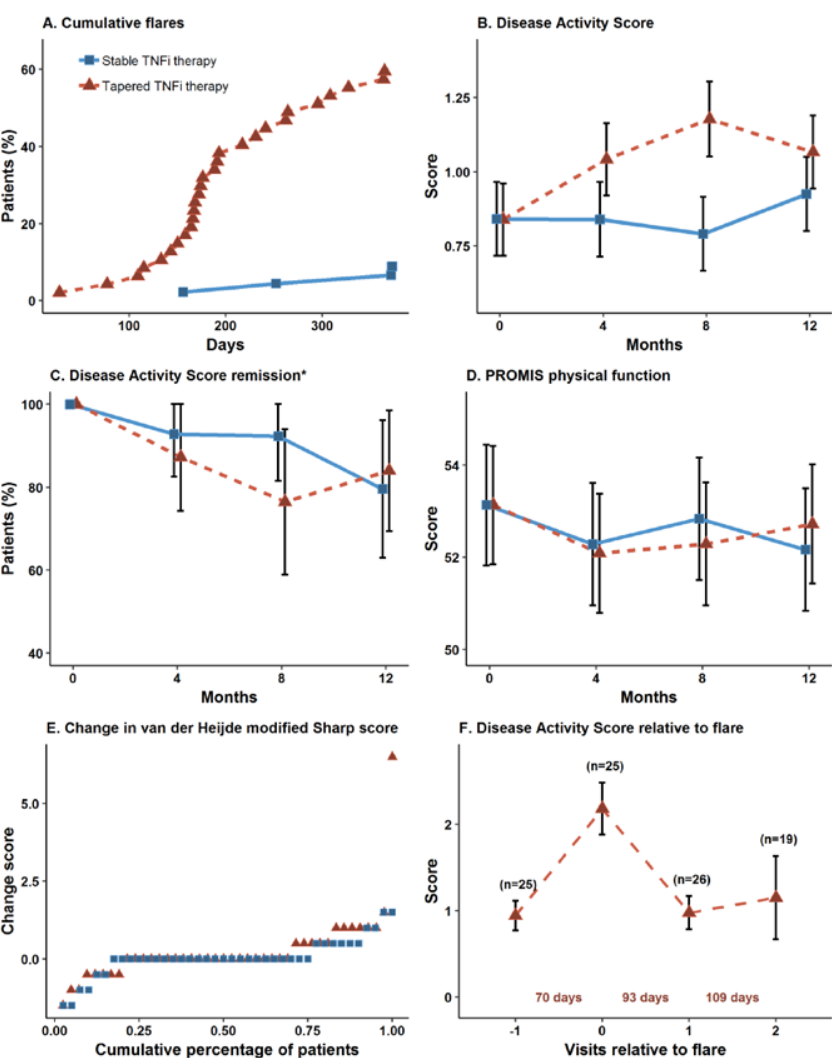

Disclosure of Interests: Siri Lillegraven: None declared, Nina Paulshus Sundlisæter: None declared, Anna-Birgitte Aga: None declared, Joe Sexton: None declared, Inge Olsen: None declared, Åse Lexberg: None declared, Tor Magne Madland: None declared, Hallvard Fremstad: None declared, Christian A. Høili Consultant of: Novartis, Gunnstein Bakland Consultant of: Novartis, UCB, Cristina Spada: None declared, Hilde Haukeland Consultant of: Novartis, Inger M. Hansen: None declared, Ellen Moholt: None declared, Till Uhlig Consultant of: Lilly, Pfizer, Speakers bureau: Grünenthal, Novartis, Daniel Solomon Grant/research support from: Funding from Abbvie and Amgen unrelated to this work, Désirée van der Heijde Consultant of: AbbVie, Amgen, Astellas, AstraZeneca, BMS, Boehringer Ingelheim, Celgene, Cyxone, Daiichi, Eisai, Eli-Lilly, Galapagos, Gilead Sciences, Inc., Glaxo-Smith-Kline Janssen, Merck, Novartis, Pfizer, Regeneron, Roche, Sanofi, Takeda, UCB Pharma; Director of Imaging Rheumatology BV, Tore K. Kvien Grant/research support from: Received grants from Abbvie, Hospira/Pfizer, MSD and Roche (not relevant for this abstract)., Consultant of: Have received personal fees from Abbvie, Biogen, BMS, Celltrion, Eli Lily, Hospira/Pfizer, MSD, Novartis, Orion Pharma, Roche, Sandoz, UCB, Sanofi and Mylan (not relevant for this abstract)., Paid instructor for: Have received personal fees from Abbvie, Biogen, BMS, Celltrion, Eli Lily, Hospira/Pfizer MSD, Novartis, Orion Pharma, Roche, Sandoz, UCB, Sanofi and Mylan (not relevant for this abstract)., Speakers bureau: Have received personal fees from Abbvie, Biogen, BMS, Celltrion, Eli Lily, Hospira/Pfizer, MSD, Novartis, Orion Pharma, Roche, Sandoz, UCB, Sanofi and Mylan (not relevant for this abstract)., Espen A Haavardsholm Grant/research support from: AbbVie, UCB Pharma, Pfizer Inc, MSD Norway, Roche Norway, Consultant of: Pfizer, AbbVie, Janssen-Cilag, Gilead, UCB Pharma, Celgene, Lilly, Paid instructor for: UCB Pharma, Speakers bureau: Pfizer, AbbVie, UCB Pharma, Celgene, Lilly, Roche, MSD

DOI: 10.1136/annrheumdis-2020-eular.1955

\section{IMPACT OF BDMARDS WITH DIFFERENT MODES OF ACTION ON FATIGUE IN RA PATIENTS}

M. Schaefer ${ }^{1}$, P. Herzer ${ }^{2}$, C. Kühne ${ }^{3}$, H. Kellner ${ }^{4}$, A. Zink ${ }^{1}$, A. Strangfeld ${ }^{1}$.

${ }^{1}$ German Rheumatism Research Centre, Epidemiology Unit, Berlin, Germany;

${ }^{2}$ Scientific Advisory Board, Munich, Germany; ${ }^{3}$ Rheumatologist, Haldensleben, Germany; ${ }^{4}$ Rheumatologist, Munich, Germany 
Table 1. Patient characteristics for different DMARD modes of action

\begin{tabular}{|c|c|c|c|c|c|c|}
\hline Parameter & csDMARDs & TNFi & RTX & ABA & IL-6 & JAKi \\
\hline $\mathrm{N}$ & 2376 & 2772 & 115 & 166 & 357 & 110 \\
\hline Fatigue at baseline & $5.9(2)$ & $6.1(2)$ & $5.9(2)$ & $5.9(1.9)$ & $6.1(2)$ & $6.3(1.9)$ \\
\hline Age [years] & $58.5(12.7)$ & $56.3(12.4)$ & $62.7(10.9)$ & $59.7(12.6)$ & $57.9(12.5)$ & $61.5(11.5)$ \\
\hline Female sex & 1809 (76.1) & 2060 (74.3) & $82(71.2)$ & $118(71)$ & $272(76.3)$ & $79(70.1)$ \\
\hline Disease duration [years] & $6.2(7.2)$ & $8.7(8.1)$ & $10.8(9.7)$ & $9.8(9.2)$ & $7.9(7.6)$ & $8.5(10)$ \\
\hline Joint erosions & 634 (28.4) & 1358 (50.5) & $62(56.8)$ & $91(55.4)$ & $158(46.4)$ & $45(41.3)$ \\
\hline Prior csDMARD therapies & $1.3(0.6)$ & $2.3(1)$ & $2.5(1.1)$ & $2.2(1)$ & $2.2(0.9)$ & $1.8(0.8)$ \\
\hline DAS28-ESR & $4.6(1.2)$ & $5(1.2)$ & $5.3(1.3)$ & $5.3(1.2)$ & $5.2(1.3)$ & $4.9(1.3)$ \\
\hline$\%$ of full physical capacity & $67.4(21.6)$ & $64.6(22)$ & $57(23.5)$ & $59.5(21.3)$ & $63.8(20.9)$ & $61.6(23)$ \\
\hline $\begin{array}{l}\text { Glucocorticoid therapy (last } \\
6 \text { months) }\end{array}$ & $1161(48.9)$ & $1747(63)$ & $76(66.4)$ & $93(56)$ & $198(55.5)$ & $42(38.1)$ \\
\hline Fibromyalgia & $73(3.1)$ & $111(4)$ & $6(5.2)$ & $7(4.2)$ & $11(3.1)$ & $1(0.9)$ \\
\hline Depression & $180(7.6)$ & $218(7.9)$ & $10(8.7)$ & $14(8.4)$ & $26(7.3)$ & $16(14.6)$ \\
\hline Ever smoker & $1252(52.7)$ & $1497(54)$ & $68(59)$ & $84(50.7)$ & $200(56)$ & 59 (53.5) \\
\hline
\end{tabular}

Results are presented as mean \pm SD or number (percentage). Absolute numbers may be rounded due to multiple imputation.

Background: Fatigue is an important patient-reported outcome. It has been reported to be potentially targetable by DMARDs with specific modes of action, particularly IL-6 inhibition [1].

Objectives: To assess to which extent patients on DMARDs with different modes of action reach fatigue levels of 2 or less on a 0 (no fatigue) to 10 (high fatigue) scale after 6 months of treatment.

Methods: The German register RABBIT is a prospective longitudinally followed cohort of RA patients enrolled with a new start of a DMARD after at least one csDMARD failure. This analysis comprises bionaive patients who were enrolled with start of a b/tsDMARD between 01/2009 and 04/2019, who had at least 1 follow-up, did not switch during the first 3 months and afterwards only within the same substance, and presented fatigue levels of $>2$ at baseline.

Poisson regression models with a robust error variance were used to calculate risk ratios (RRs) for reaching fatigue values $\leq 2$, for all DMARD modes of action. Propensity score weighting was used to adjust for confounding by indication. Multiple imputation of missing values was performed.

Results: Baseline fatigue levels were 5.1 overall and 6.1 among patients with a fatigue level of $>2$ points on average. They were comparable among different DMARD modes of action. csDMARD patients had lower values than others regarding disease duration, disease activity, or joint erosions (Table 1).

The RR of IL-6 inhibitors for achieving a fatigue level of $\leq 2$ was $1.34(95 \% \mathrm{Cl}$ : 1.09 - 1.64) compared to csDMARDs. Among other factors, current smoking, prevalent fibromyalgia and depression had a negative impact on achieving a low fatigue level (Table 2).

Table 2. Risk ratios for achieving fatigue levels $\leq 2$

\begin{tabular}{|c|c|c|c|}
\hline \multirow{2}{*}{$\frac{\text { Parameter (at baseline) }}{\text { Fatigue (1 point higher) }}$} & \multirow{2}{*}{$\begin{array}{l}\mathrm{RR} \\
\mathbf{0 . 8 3}\end{array}$} & \multicolumn{2}{|c|}{$95 \%$ confidence interval } \\
\hline & & $(0.80$ & $0.86)$ \\
\hline TNF inhibitor (vs. csDMARDs) & 1.11 & (0.99; & 1.24) \\
\hline Rituximab (vs. csDMARDs) & 1.10 & $(0.71$ & 1.68) \\
\hline Abatacept (vs. csDMARDs) & 1.13 & $(0.82$ & 1.54) \\
\hline IL-6 inhibitor (vs. csDMARDs) & 1.34 & (1.09; & 1.64) \\
\hline JAK inhibitor (vs. csDMARDs) & 1.19 & $(0.81$ & $1.75)$ \\
\hline Age (5 years more) & 0.97 & $(0.95$ & $0.99)$ \\
\hline Female sex & 0.83 & $(0.74$ & $0.92)$ \\
\hline Patient global health (1 point higher) & 0.97 & $(0.94$ & $0.997)$ \\
\hline Joint erosions & 1.19 & $(1.07$ & 1.32) \\
\hline Current smoking & 0.86 & $(0.76$ & $0.98)$ \\
\hline Former smoking & 0.92 & $(0.82$ & $1.04)$ \\
\hline Fibromyalgia & 0.56 & (0.35; & $0.90)$ \\
\hline Depression & 0.75 & (0.59; & $0.95)$ \\
\hline
\end{tabular}

Conclusion: Treatment with IL-6 inhibitors significantly increases the chance of reaching low fatigue levels within half a year in RA patients, while current smoking reduces it.

References:

[1] Choy E.H.S. and Calabrese L. H Rheumatology 2018;57:1885-95.

Acknowledgments: RABBIT is supported by a joint, unconditional grant from AbbVie, Amgen, BMS, Fresenius Kabi, Hexal, Lilly, MSD, Mylan, Pfizer, Roche, Samsung Bioepis, Sanofi-Aventis, and UCB.

Disclosure of Interests: Martin Schaefer: None declared, Peter Herzer Speakers bureau: AbbVie, Novartis, Sanofi, Janssen, Cornelia Kühne Grant/ research support from: Novartis, Amgen, Roche/Chugai, Pfizer, Celgene, AbbVie, Sanofi, Herbert Kellner Grant/research support from: Biogen, Consultant of: Biogen, Speakers bureau: Biogen, Angela Zink Speakers bureau: AbbVie, Amgen, BMS, Gilead, Hexal, Janssen, Lilly, MSD, Pfizer, Roche, Sanofi Aventis, UCB, Anja Strangfeld Speakers bureau: AbbVie, BMS, Pfizer, Roche, Sanofi-Aventis

DOI: 10.1136/annrheumdis-2020-eular.1498

\section{OP0021 \\ OLOKIZUMAB, MONOCLONAL ANTIBODY AGAINST IL6, IN PATIENTS WITH MODERATELY TO SEVERELY ACTIVE RHEUMATOID ARTHRITIS INADEQUATELY CONTROLLED BY METHOTREXATE: EFFICACY AND SAFETY RESULTS OF PHASE III CREDO-1 STUDY}

E. Nasonov ${ }^{1}$, R. Stoilov ${ }^{2}$, T. Tyabut ${ }^{3}$, M. C. Genovese ${ }^{4}$ on behalf of Saeed Fatenejad (United States of America), Diana Krechikova, Elena Korneva, Alexey Maslyansky, Tatiana Plaksina, Marina Stanislav, Sergey Yakushin, Elena Zonova (Russian Federation). ${ }^{1}$ SRI of Rheumatology n.a.V.A.Nasonova, Moscow, Russian Federation; ' ${ }^{2}$ University Hospital "St. Ivan Rilski", Sofia, Bulgaria; ${ }^{3}$ Belarusian Medical Academy of Postgraduate Education, Minsk, Belarus; ${ }^{4}$ Stanford University, Stanford, United States of America

Background: Olokizumab (OKZ) is a new humanized monoclonal antibody targeting IL-6 ${ }^{1,2}$. Here we present the results of the first phase III study of OKZ in patients with Rheumatoid Arthritis (RA).

Objectives: The primary objective was to evaluate the safety and efficacy of OKZ administered subcutaneously (SC) every 2 weeks (q2w) and 64mg SC every 4 weeks ( $q 4 w$ ) vs placebo in the treatment moderate-to-severe RA.

Methods: In this randomized, double-blind, placebo-controlled, multicenter study in patients with moderately to severely active RA despite methotrexate (MTX) (ClinicalTrials.gov Identifier NCT02760368, CREDO-1) patients received SC injections of OKZ $64 \mathrm{mg}$ q2w, OKZ $64 \mathrm{mg}$ q4w and PBO q2w for 24 weeks. Starting at Week (Wk) 14, non-responders were rescued. After Wk 24, subjects were eligible for an open-label study extension.

The primary endpoint was American College of Rheumatology $20 \%$ (ACR20) response at Wk 12.

Secondary endpoints included: percentage of subjects with low disease activity, improvement of physical ability. Safety outcomes, including treatment-emergen adverse events (TEAEs), treatment-emergent serious adverse events (TESAEs) and laboratory abnormalities (via central lab) were assessed.

Results: 428 patients were randomized to OKZ 64mg q2w $(n=143)$, OKZ 64mg q4w $(n=142)$, and PBO ( $n=143)$. Baseline characteristics were comparable between treatment arms: mean (SD) age was 51.3 (12.0) years, $82.7 \%$ of patients were female, disease duration 8.14 (7.6) and all patients were on MTX. Mean (SD) baseline DAS28-CRP was 6.0 (0.76) and HAQ-DI was $1.72(0.49) .130(90.9 \%)$ patients in q2w, 134 (94.4\%) in q4w and 132 (92.3\%) in PBO arms completed the study. Both regimens of OKZ were significantly better than PBO in all primary and secondary endpoints (Table 1).

Table 1. Key efficacy results (intent-to-treat population) NRI ${ }^{1}$

\begin{tabular}{|c|c|c|c|}
\hline & $O K Z$ q2w & $O K Z q 4 w$ & PBO \\
\hline Number of Subjects & 143 & 142 & 143 \\
\hline $\begin{array}{l}\text { ACR20 Response, } n \text { (\%) Wk } 12 \text { (primary } \\
\text { endpoint) }\end{array}$ & $91(63.6)^{\star \star \star}$ & $100(70.4)^{\star \star \star}$ & $37(25.9)$ \\
\hline DAS28<3.2 Response, n (\%) Wk 12 & $48(33.6)^{\star \star \star}$ & $55(38.7)^{\star \star \star}$ & $5(3.5)$ \\
\hline HAQ-DI Wk 12 LSM (SE) & $-0.54(0.041)$ & $-0.56(0.042)$ & $-0.20(0.042)$ \\
\hline Treatment Comparison vs PBO LSM & $-0.34^{\star \star \star}(0.059)$ & $-0.36^{\star \star \star}(0.059)$ & \\
\hline \multicolumn{4}{|l|}{ Difference (SE) } \\
\hline $97.5 \% \mathrm{Cl}$ for LSM Difference & $-0.47,-0.21$ & $-0.49,-0.23$ & \\
\hline ACR50 Response, n (\%) Wk 24 & $61(42.7)^{\star \star \star}$ & $69(48.6)^{\star \star \star}$ & $11(7.7)$ \\
\hline CDAI $\leq 2.8$ Response, $n(\%)$ Wk 24 & $12(8.4)^{\star \star}$ & $11(7.7)^{\star \star}$ & 0 \\
\hline
\end{tabular}

- NRI, Non-Responder Imputation; LSM, Least Squares Mean; ${ }^{* *} \mathrm{p}$-value difference from $\mathrm{PBO}<0.001 ;{ }^{* \star *} \mathrm{p}$-value difference from PBO $<0.0001 ;$ SE, Standard Error

The key efficacy outcomes were maintained throughout the 24-week.

Overall incidence of TEAEs was $58.0 \%$ in OKZ q2w arm; $57.0 \%$ in OKZ q4w arm and $43.7 \%$ in PBO, TEAEs leading to study treatment discontinuation were reported in $4.9 \%, 3.5 \%$ and $0.7 \%$ patients, respectively. 